normal glucose and protein. Following withdrawal of $6 \mathrm{ml} \mathrm{CSF}$, the rigidity and neck pains resolved and neck movements were normal. Papilledema was reduced after 3 weeks treatment with acetazolamide and dexamethasone. Patient 2, a 9year-old previously healthy female had a 10 day history of neck pain followed by headache, neck stiffness, and papilledema. CT was normal and CSF pressure 280 $\mathrm{mm}$. Within one half hour of removal of $7 \mathrm{ml} \mathrm{CSF}$, symptoms were completely relieved. Symptoms did not recur during subsequent treatment with acetazolamide and dexamethasone. Patient 3 , an 8-year-old male was admitted with a 6 week history of headaches, torticollis for 4 weeks, right sided neck pain and papilledema. Known causes of pseudotumor, including trauma, infection, vitamins, and endocrine factors, were absent. CT showed slit-like ventricles. CSF pressure was $480 \mathrm{~mm}$. Torticollis and neck pain resolved within 1 hour after lumbar puncture. He was asymptomatic and had normal fundi at 3 week follow-up, following therapy with acetazolamide and prednisone. (Straussberg R, Harel L, Amir J. Pseudotumor cerebri manifesting as stiff neck and torticollis. Pediatr Neurol March 2002;26:225-227). (Respond: Dr Straussberg, Department of Pediatrics C, Schneider Children's Miedical Center of Israel, Petah Tikva, Israel 49202).

COMMENT. Pseudotumor cerebri should be considered in the differential diagnosis of acute onset of stiff neck or torticollis. A recent review of 10 children with pseudotumor cerebri, cited by the authors, found 4 patients presenting with stiff neck (Cinciripini GS et al. Am I Ophthalmol 1999;127:178-182). The mechanism of the stiff neck and torticollis and the association with prepubertal cases of pseudotumor are undetermined. The more classical presenting manifestations are headache, vomiting, and papilledema. Other more common neurologic disorders that underly neck rigidity and pain and/or torticollis are cervical trauma or inflammation, meningitis, subarachnoid hemorrhage, posterior fossa tumor, spinal cord syrinx, and cervical radiculitis. Funduscopic examination for papilledema is recommended in children presenting with unexplained neck rigidity or torticollis, with or without headache. The rapid relief of symptoms following lumbar puncture and the resolution of papilledema following a short course of acetazolamide and steroids are noteworthy.

\title{
MILLER FISHER SYNDROME PRESENTING AS PSEUDOTUMOR
}

Two female children, ages 9 and 2 years, who presented with pseudotumor cerebri and within 2 to 3 days, were diagnosed with Miller Fisher syndrome are reported from the Hopital Universitaire des Enfants Reine Fabiola, Brussels, Belgium. The 9-year old had sudden onset of frontal headaches associated with nausea, diplopia, and convergent stabismus. Two weeks previously she had developed an acute febrile illness with diarrhea. Neurologic examination on admission was normal except for a left VIth nerve palsy. CT was normal, but opening pressure on lumbar puncture was $300 \mathrm{~mm}$ water. Headache and nausea improved initially after removal of $15 \mathrm{ml} \mathrm{CSF}$, but then recurred, with vomiting. Following treatment with acetazolamide, symptoms again improved, but 3 days later, she developed Miller Fisher syndrome, presenting with bilateral III and VI nerve palsies, ataxia, and areflexia. EMG and NCS were normal, and $\mathrm{H}$ reflexes were absent, indicating impaired nerve conduction in proximal fibers and acute demyelinating polyneuropathy. Plasma serology was positive for Campylobacter jejuni and anti-GQ1b antiganglioside antibodies. Treatment with a 2-day course of iv immunoglobulin resulted in lessening of ataxia in 1 week, and recovery of reflexes and eye movements in one month and at 3 month follow-up. The 2-year old patient had a similar history and diagnoses. Plasma serology was negative for $C$ jejuni, but positive for antimyelin antibodies. EMG and NCS were abnormal, with 
widespread fibrillations, reduced motor and sensory nerve conduction velocities, conduction blocks, and increased $\mathrm{F}$ wave latencies, indicative of demyelination and also signs of axonal neuropathy. Following iv immunoglobulin and corticosteroids, dysarthria and dysphagia had resolved and ataxia was mild at 4week follow-up. (Mewasingh LD, Sekhara T, Dachy B et al. Benign intracranial hypertension: atypical presentation of Miller Fisher syndrome? Pediatr Neurol March 2002:26:228-230). (Respond: Professor Bernard Dan MD, Department of Neurology, Hopital Universitaire des Enfants Reine Fabiola, 15 Avenue JJ Crocq, 1020 Brussels, Belgium).

COMMENT. The syndrome first described by Dr Miller Fisher, neurologist at the Massachusetts General Hospital, is "An unusual variant of acute idiopathic polyneuritis (syndrome of ophthalmoplegia, ataxia and areflexia)." (N Engl I Med 1956;255:57-65). The immune-mediated, acute demyelinating Guillain-Barre variant involves anti-GQ1b antiganglioside antibodies in $90 \%$ of cases, and is preceded by a respiratory or gastrointestinal infection in two thirds, Campylobacter jejuni being a common isolate in the cases that present with diarrhea. The cases in the present report are particularily rare since they occur in children, and the MF syndrome is preceded by symptoms of pseudotumor cerebri. The co-occurrence of papilledema and Guillain-Barre syndrome is reported in 4\% of childhood cases (Peterman AF et al. Neurology 1959;9:533-539). The authors cite only one previous case of MF syndrome, in a 5-year-old male, presenting with pseudotumor (Macaya A et al. Pediatr Neurol 1988;4:120-121). CSF opening pressure and funduscopic examination should be included in the evaluation of patients with Guillain-Barre syndrome.

\section{INFECTIOUS DISORDERS}

RISK OF NEUROLOGIC SEQUELAE WITH BACTERIAL MENINGITIS Predictors of permanent neurologic sequelae or death following bacterial meningitis were determined in a case-controlled study at Sophia Children's Hospital, Rotterdam, The Netherlands. The study population of 93 children presenting with meningeal signs included 23 cases with neurologic sequelae and 70 controls without sequelae ( $52 \%$ boys, median age $2.8 \mathrm{yrs}$ ). Of the 23 cases, 2 died (8.7\%), 6 were deaf $(26.1 \%), 8$ had mild hearing loss (34.7\%), 4 were severely retarded (17.4\%) and 3 had epilepsy, mild iocomotor deficits or mild mental retardation $(13 \%)$. Significant independent predictors for an adverse outcome were maie gender, the occurrence of atypical convulsions before admission (duration $>15 \mathrm{~min}$, non-generalized jerks, incomplete recovery, multiple convulsions within $24 \mathrm{hr}$ ), low body temperature at admission, and especially, the pathogen type Streptococcus pneumoniae. The area under the ROC curve (estimated predictive performance) of this prediction rule was 0.87 (95\% confidence interval: 0.78-0.96). A risk score computed for each patient by assigning points for each predictor present was used to classify patients into adverse outcome categories. (Oostenbrink R, Moons KGM, Derksen-Lubsen G, Grobbee DE, Moll HA. Early prediction of neurological sequelae or death after bacterial meningitis. Acta Paediatr 2002;91:391-398). (Respond: Dr R Oostenbrink, Sophia Children's Hospital, Room Sp 1545, Dr Molewaterplein 60, NL-3015 GJ Rotterdam, The Netherlands).

COMMENT. Permanent neurological sequelae or death after bacterial meningitis in childhood may be predicted from the early clinical characteristics. 